\title{
SCALING LAWS FOR LASER WAKEFIELD ACCELERATORS*
}

\author{
E. Esarey $^{\dagger}$ and W.P. Leemans \\ Lawrence Berkeley National Laboratory, University of California, Berkeley CA 94720
}

\section{Abstract}

Scaling laws are derived as a function of laser wavelength for various quantities of interest for a single stage of a channel-guided laser wakefield accelerator. Comparisons are made for drive laser pulses of equal energies. Scalings that favor short or long wavelengths depend on the quantity that is to be optimized. For example, holding the number of electrons per bunch constant favors shorter wavelengths, whereas holding the energy gain constant can favor longer wavelengths.

\section{INTRODUCTION}

Plasma-based accelerators [1] are capable of sustaining ultrahigh electric fields $E_{z}$ on the order of $E_{0}=c m \omega_{p} / e$, i.e.,

$$
E_{0}[\mathrm{~V} / \mathrm{m}] \simeq 96 n^{1 / 2}\left[\mathrm{~cm}^{-3}\right],
$$

where $n$ is the ambient plasma density and $\omega_{p}=$ $\left(4 \pi n e^{2} / m\right)^{1 / 2}$ is the plasma frequency. Although the accelerating field is very high, the wavelength of the accelerating structure (i.e., the plasma wave) is very short and on the order of the plasma wavelength $\lambda_{p}=2 \pi c / \omega_{p}$, i.e.,

$$
\lambda_{p}[\mu \mathrm{m}] \simeq 3.3 \times 10^{10} n^{-1 / 2}\left[\mathrm{~cm}^{-3}\right],
$$

e.g., $E_{0} \sim 30 \mathrm{GV} / \mathrm{m}$ and $\lambda_{p} \simeq 100 \mu \mathrm{m}$ for $n \simeq 10^{17}$ $\mathrm{cm}^{-3}$. The shortness of $\lambda_{p}$ has important consequences. For example, if an electron bunch is to be injected into the plasma wave such that its initial low energy spread is maintained, then it is desirable for the initial bunch length $L_{b}$ to be short compared to $\lambda_{p}$. Furthermore, the bunch must be injected at the optimal plasma wave phase with femtosecond timing accuracy. These requirements are beyond the state-of-the-art for conventional injector technology (e.g., photo-injectors). Conversely, the shortness of $\lambda_{p}$ and the wide-spread availability of ultrashort high power lasers may allow plasma-based accelerators to be developed as a compact source of ultrashort, high energy electron bunches [2-7].

In the standard laser wakefield accelerator (LWFA) a single, intense, short (a pulse length $L \sim \lambda_{p}$ ) is used to drive the wakefield. As the pulse propagates through the plasma, its ponderomotive force $F_{p} \propto \nabla a^{2}$ expells plasma electrons, thus exciting a large amplitude plasma wave with phase veloctiy $v_{p} \simeq c$ in its wake. Here, $a^{2}$ is proportional to the laser intensity $I$,

$$
a^{2} \simeq 7.2 \times 10^{-19} \lambda^{2}[\mu \mathrm{m}] I\left[\mathrm{~W} / \mathrm{cm}^{2}\right],
$$

* Work supported by the U.S. Department of Energy under contract No. DE-AC-03-76SF0098.

† Email: ehesarey@1bl.gov

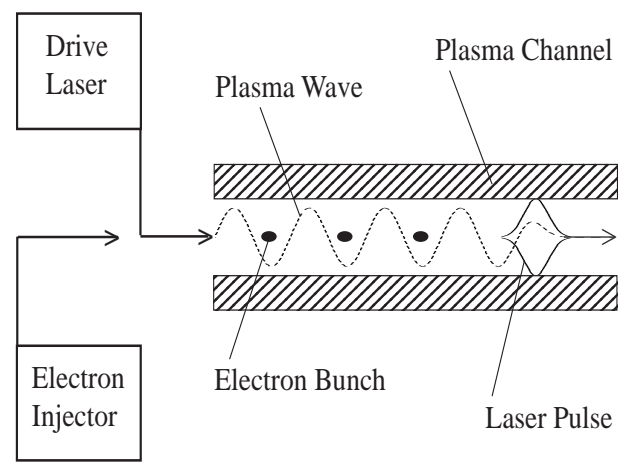

Figure 1: Schematic of a channel-guided LWFA. The plasma channel guides the laser pulse and supports the wakefield, which is generated behind the laser pulse with a phase velocity $v_{p} \simeq c$. Short electron bunches with length $L_{b} \ll \lambda_{p}$ are injected into the wakefield for acceleration to high energy.

and is related to the power $P$ of a Gaussian radial intensity profile $I \propto \exp \left(-2 r^{2} / r_{s}^{2}\right)$ by $P[\mathrm{GW}] \simeq 21.5 a^{2} r_{s}^{2} / \lambda^{2}$, where $\lambda$ is the laser wavelength, $r_{s}$ is the spot size, and linear polarization has been assumed.

A practical high-energy LWFA requires the use of a plasma channel to guide the laser pulse (see Fig. 1). In vacuum, laser propagation is typically limited by diffraction, the characteristic distance of which is the Rayleigh length $Z_{R}=\pi r_{0}^{2} / \lambda$, where $r_{0}$ is the laser spot radius at focus (e.g., $Z_{R} \simeq 3 \mathrm{~mm}$ for $r_{0}=30 \mu \mathrm{m}$ and $\lambda=1 \mu \mathrm{m}$ ). Extending the single-stage interaction (acceleration) length beyond $Z_{R}$ requires optical guiding [8]. In the standard LWFA, this can be achieved with a preformed plasma density channel [8-15]. The index of refraction in a plasma is approximately $\eta_{R} \simeq 1-\lambda^{2} / 2 \lambda_{p}^{2}$. As in an optical fiber, a plasma channel can provide optical guiding if the index of refraction peaks on axis, $\partial \eta_{R} / \partial r<0$, which can be achieved with a plasma density profile that has a local minimum on axis, $\partial n / \partial r>0$. Specifically, a channel with a radially parabolic density profile of the form $n(r)=n_{0}+\Delta n r^{2} / r_{0}^{2}$ can guide a Gaussian laser pulse with a constant spot size $r_{0}$ provided the channel depth $\Delta n$ satisfies $\Delta n=\Delta n_{c}$, where $\Delta n_{c}=1 / \pi r_{e} r_{0}^{2}$ is the crit- 
ical channel depth [8] and $r_{e}=e^{2} / m_{e} c^{2}$ is the classical electron radius, i.e.,

$$
\Delta n_{e}\left[\mathrm{~cm}^{-3}\right] \simeq 1.13 \times 10^{20} / r_{0}^{2}[\mu \mathrm{m}]
$$

The following discussion will be restricted to standard LWFAs that utilize channel guiding.

The most common sources of high power, short laser pulses are solid-state systems based on the technique of chirped-pulse amplification (CPA) [16]. Such CPA systems typically have $\lambda \sim 1 \mu \mathrm{m}$ and have been used in numerous LWFA and channel-guiding experiments [9-15]. Alternatively, high-power, short-pulse $\mathrm{CO}_{2}$ laser systems ( $\lambda \sim 10 \mu \mathrm{m}$ ) are being developed and will be available for future LWFA experiments [17]. In this paper, scaling laws with respect to $\lambda$ for relevant LWFA quantities are derived and discussed.

\section{SCALING LAWS}

In this section, simple scaling laws for LWFA quantities are presented under idealized assumptions. These idealized scaling laws assume the following:

1. A standard LWFA that is channel guided.

2. The mildly relativistic regime, $a^{2} \ll 1$.

3. The acceleration length is limited by electron dephasing.

4. The plasma channel is sufficiently broad such that the formula describing wakefield generation in a uniform plasma apply.

5. The transverse size of the laser pulse is $2 c / \omega_{p}$ and the transverse size of the electron bunch is $c / \omega_{p}$.

6. The total electrons per bunch is the beam loading limit.

In the following, when equations are presented in practical form (with numerical coefficients), $E_{z}$ is in $\mathrm{V} / \mathrm{m}, n$ is in $\mathrm{cm}^{-3}, \lambda$ is in $\mu \mathrm{m}, I$ is in $\mathrm{W} / \mathrm{cm}^{2}, W_{L}$ is in $\mathrm{J}, L_{d}$ is in $\mathrm{m}$, $\Delta W$ is in $\mathrm{GeV}, \operatorname{Lum}_{s}$ is in $\mathrm{cm}^{-2}$, and $a^{2}$ is dimensionless.

In the mildly relativistic limit within a broad channel, the axial electric field of the wake [1] can be written as $E_{z}=0.38 a^{2} E_{0}$, where $E_{0}=m_{e} c \omega_{p} / e=96 n^{1 / 2}$, i.e.,

$$
\begin{aligned}
E_{z} & =2.7 \times 10^{-17} I \lambda^{2} n^{1 / 2} \\
& =3.4 \times 10^{-25} W_{L} \lambda^{2} n^{2}
\end{aligned}
$$

This assumes a linearly polarized laser pulse with Gaussian profiles in the radial and axial directions. This also assumes that the laser pulse length is optimized to maximize the wakefield amplitude, i.e., $L=\lambda_{p} / \sqrt{2 \pi}=0.4 \lambda_{p}$, where the pulse length $L$ is defined such that $W_{L}=$ $(1 / 8 \pi) A_{L} E_{L}^{2} L$ is the pulse energy, $\lambda_{p}=2 \pi c / \omega_{p}$ is the plasma wavelength, $E_{L}$ is the peak laser electric field,
$A_{L}=\pi r_{0}^{2} / 2$ is the cross-sectional area of the Gaussian pulse, and $r_{0}$ is the laser spot size.

The laser spot size is assumed to be $r_{0}=2 c / \omega_{p}$ in order to ensure high efficiency of energy transfer between the wake and the accelerated electrons [18], since electrons loaded near the axis will absorb wake energy out to a radius of approximately $c / \omega_{p}$. Furthermore,

$$
a^{2}=9.4 \times 10^{-27} W_{L} \lambda^{2} n^{3 / 2}
$$

The acceleration length is assumed to be equal to the electron dephasing length $L_{D}=\lambda_{p}^{2} / \lambda^{2}$,

$$
L_{d}=3.7 \times 10^{25} \lambda^{-2} n^{-3 / 2}
$$

The ideal maximum energy gain is given by $\Delta W=$ $e E_{z} L_{d}$,

$$
\Delta W=I / n=1.3 \times 10^{-8} W_{L} n^{1 / 2}
$$

The number of electrons accelerated per bunch is assumed to be equal to the beam loading limit [18] $N_{b}=$ $E_{z} A_{b} / 4 \pi e$, where $A_{b}$ is the effective cross-sectional area of the beam which is assumed to be $A_{b}=\pi c^{2} / \omega_{p}^{2}$,

$$
N_{b}=1.7 \times 10^{-9} W_{L} \lambda^{2} n
$$

Another figure of merit is the luminosity $\mathrm{Lum}=$ $\left(k_{b} f_{b} / 4 \pi\right) N_{b}^{2} / \sigma_{x} \sigma_{y}$, where $k_{b}$ is the number of bunches per linac, $f_{b}$ is the linac rep rate, and $\sigma_{x, y}$ are the transverse rms bunch sizes, which are assumed to be equal to $c / \omega_{p}$. For scaling purposes, it is convenient to define the "single bunch" luminosity as $\operatorname{Lum}_{s}=N_{b}^{2} / \sigma_{x} \sigma_{y}$,

$$
\operatorname{Lum}_{s}=9.9 \times 10^{-30} W_{L}^{2} \lambda^{4} n^{3}
$$

\section{EXAMPLES}

Next, to determine scaling with wavelength, several examples are given. In all these examples, the laser pulse energy $W_{L}$ is assumed constant.

\subsection{Constant $E_{z}$}

The axial electric field of the wake is held fixed (in addition to the pulse energy). This implies:

$$
\begin{gathered}
n \propto \lambda^{-1}, L_{d} \propto \lambda^{-1 / 2}, \Delta W \propto \lambda^{-1 / 2}, \\
N_{b} \propto \lambda, \operatorname{Lum}_{s} \propto \lambda
\end{gathered}
$$

\subsection{Constant $L_{d}$}

The acceleration length is held fixed (in addition to the pulse energy). This implies:

$$
\begin{gathered}
n \propto \lambda^{-4 / 3}, E_{z} \propto \lambda^{-2 / 3}, \Delta W \propto \lambda^{-2 / 3}, \\
N_{b} \propto \lambda^{2 / 3}, L_{u m_{s}} \propto \text { constant }
\end{gathered}
$$




\subsection{Constant $\Delta W$}

The electron energy gain is held fixed (in addition to the pulse energy). This implies:

$$
\begin{gathered}
n \propto \text { constant }, E_{z} \propto \lambda^{2}, L_{d} \propto \lambda^{-2}, \\
N_{b} \propto \lambda^{2}, \operatorname{Lum}_{s} \propto \lambda^{4}
\end{gathered}
$$

\subsection{Constant $N_{b}$}

The number of electrons per bunch is held fixed (in addition to the pulse energy). This implies:

$$
\begin{gathered}
n \propto \lambda^{-2}, E_{z} \propto \lambda^{-2}, L_{d} \propto \lambda, \\
\Delta W \propto \lambda^{-1}, \text { Lum }_{s} \propto \lambda^{-2}
\end{gathered}
$$

\subsection{Constant Lum $_{s}$}

The single bunch luminosity is held fixed (in addition to the pulse energy). This implies:

$$
\begin{gathered}
n \propto \lambda^{-4 / 3}, E_{z} \propto \lambda^{-2 / 3}, L_{d} \propto \text { constant }, \\
\Delta W \propto \lambda^{-2 / 3}, N_{b} \propto \lambda^{2 / 3}
\end{gathered}
$$

\section{DISCUSSION}

In making comparisons between $\lambda=1 \mu \mathrm{m}$ and $\lambda=10 \mu \mathrm{m}$ drivers, care must be taken so as not to violate the above assumptions, in particular, $a^{2} \ll 1$. Note that $a^{2} \propto$ $W_{L} \lambda^{2} n^{2 / 3}$. Hence, when making comparisons at constant density and pulse energy, as in Case 3.3, the assumption $a^{2} \ll 1$ may be violated at long wavelengths. On the other hand, for short wavelengths, operation at high density is valid.

A definitive conclusion regarding an optimum driver wavelength is problematic. For example, at sufficiently low density (such that $a^{2} \ll 1$ ), a design for a fixed energy gain favors longer wavelengths, as implied by Case 3.3. On the other hand, a design for a fixed number of electrons per bunch favors short wavelengths, as implied by Case 3.4. Furthermore, a design for a fixed acceleration distance (and fixed luminosity) allows higher energies to be obtained for short wavelengths, however, a higher bunch number is obtained for long wavelengths. The above scaling laws all assume a fixed laser pulse energy. A rigorous study of a LWFA for various wavelength drivers must also include other properties of the driver, such as repetition rate, pulse stability, and average power. Since laser technology is rapidly progressing, a rigorous design study is premature. In terms of physics experiments, invaluable information can be obtained at both 1 and 10 micron.

\section{ACKNOWLEDGMENTS}

The authors acknowledge useful conversations with I.V. Pogorelsky, T. Katsouleas, and C. Joshi.

\section{REFERENCES}

[1] For a review, see E. Esarey, P. Sprangle, J. Krall, and A. Ting, IEEE Trans. Plasma Sci. 24, 252 (1996).

[2] D. Umstadter, J.K. Kim, and E. Dodd, Phys. Rev. Lett. 76, 2073 (1996).

[3] R.G. Hemker, K.C. Tzeng, W.B. Mori, C.E. Clayton, and T. Katsouleas, Phys. Rev. E 57, 5920 (1998).

[4] E. Esarey, R.F. Hubbard, W.P. Leemans, A. Ting, and P. Sprangle, Phys. Rev. Lett. 79, 2682 (1997).

[5] W.P. Leemans, P. Volfbeyn, K.Z. Guo, S. Chattopadhyay, C.B. Schroeder, B.A. Shadwick, P.B. Lee, J.S. Wurtele, and E. Esarey, Phys. Plasmas 5, 1615 (1998).

[6] C.B. Schroeder, P.B. Lee, J.S. Wurtele, E. Esarey, and W.P. Leemans, Phys. Rev. E, May (1999).

[7] E. Esarey, C.B. Schroeder, and W.P. Leemans, Phys. Plasmas, May (1999).

[8] For a review, see E. Esarey, P. Sprangle, J. Krall, and A. Ting, IEEE J. Quant. Elect. 33, 1879 (1997).

[9] H.M. Milchberg, T.R. Clark, C.G. Durfee, T.M. Antonsen, and P. Mora, Phys. Plasmas 3, 2149 (1996).

[10] V. Malka, E. de Wispelaere, F. Amiranoff, S. Baton, R. Bonadio, C. Coulaud, R. Haroutunian, A. Modena, D. Puissant, C. Stenz, S. Muller, and M. Casanova, Phys. Rev. Lett. 79, 2979 (1997).

[11] T. Ditmire, R.A. Smith, and M.H.R. Hutchinson, Opt. Lett. 23, 322 (1998).

[12] P. Volfbeyn and W.P. Leemans, in Proc. 6th European Part. Accel. Conf., Ed. by S. Myers, L. Liljeby, Ch. Petit-JeanGenaz, J. Poole, and K.G. Rensfelt (Inst. of Phys., Philadelphia, 1998), p. 265; P. Volfbeyn, MIT Ph.D. Thesis, LBNL Report 41892 (1998).

[13] E. Gaul, S.P. Le Blanc, and M.C. Downer, Advanced Accelerator Concepts, Edited by W. Lawson, AIP Conf. Proc. (AIP, NY, in press).

[14] P.S. Volfbeyn, E. Esarey, and W.P. Leemans, Phys. Plasmas, May (1999).

[15] Y. Ehrlich, C. Cohen, A. Zigler, J. Krall, P. Sprangle, and E. Esarey, Phys. Rev. Lett. 77, 4186 (1996); Y. Ehrlich, C. Cohen, D. Kaganovich, A. Zigler, R.F. Hubbard, P. Sprangle, and E. Esarey, J. Opt. Soc. Am. B 15, 2416 (1998).

[16] G.A. Mourou, C.P.J. Barty, and M.D. Perry, Physics Today 51, 22 (1998).

[17] I.V. Pogorelsky, Nucl. Instr. Meth. Phys. A 410524 (1998); I.V. Pogorelsky et al., in Advanced Accelerator Concepts. edited by W. Lawson, AIP Conf. Proc. (Amer. Inst. Phys., $\mathrm{NY}$, in press).

[18] T. Katsouleas, S. Wilks, P. Chen, J.M. Dawson, and J.J. Su, Particle Accelerators 22, 81 (1987). 